\title{
P6 acupoint stimulation for prevention of postoperative nausea and vomiting in patients undergoing craniotomy: study protocol for a randomized controlled trial
}

\author{
Jian-qin Lv, Rui-zhi Feng and Ning Li
}

\begin{abstract}
Background: The incidence of postoperative nausea and vomiting (PONV) is 50 to $79 \%$ after neurosurgery. Our study is designed to evaluate the efficacy of pericardium 6 (P6; also known as Neiguan) acupoint stimulation versus placebo, and versus routine antiemetic for the prevention of PONV after craniotomy, as well as to compare the efficacy of invasive acupuncture with non-invasive transcutaneous electrical nerve stimulation (TENS) on P6.

Methods/design: This is a single-center, prospective, double-blind, five-arm, parallel-group, randomized controlled trial (RCT). All groups will be given routine intravenous ondansetron $8 \mathrm{mg}$ administered before skin closure. Upon regaining consciousness from general anaesthesia, patients will receive one of five interventions: 1) P6 acupuncture bilaterally for 30 minutes, stimulated every 10 minutes to keep de qi sensation; 2) sham acupuncture bilaterally for 30 minutes with no stimulation; 3) P6 stimulation via active TENS electrodes bilaterally for 30 minutes, with stimulation frequency and intensity set to when de qi sensation is felt; 4) sham P6 stimulation via inactive TENS electrode bilaterally for 30 minutes; and 5) usual practice of pharmacological emesis prevention. The incidence of postoperative vomiting during the first 24 hours is the main outcome. Secondary outcomes include: complete response rate, severity of nausea, total rescue metoclopramide dose used and patient satisfaction with PONV management.
\end{abstract}

Discussion: The results from this study could potentially confirm that P6 acupoint stimulation is an effective adjunct to standard antiemetic drug therapy for the prevention of PONV in patients undergoing craniotomy. Our study may also confirm that conventional acupuncture is more effective than TENS.

Trial registration: This study is registered with the Chinese Clinical Trial Registry: ChiCTR-TRC-13003026.

Keywords: Acupoint stimulation, Nausea, Vomiting, Craniotomy

\section{Background}

As a common complication of anesthesia and surgery, postoperative nausea and vomiting (PONV) remains problematic despite use of antiemetics. PONV delays the patient's recovery from anesthesia, extends their hospital stay and increases overall healthcare costs [1]. Although PONV is rarely fatal, the physical act of vomiting may increase cerebral intravascular pressure or intracranial pressure, which may lead to cause severe consequences in

\footnotetext{
* Correspondence: zhenjiuhuaxi@163.com

Department of Integrated Traditional and Western Medicine, West China Hospital of Si-chuan University, Chengdu 610041, China
}

patients who have undergone craniotomy. Studies have reported an average incidence of PONV of up to 38.3\% following common surgeries [2], while in patients specifically undergoing neurosurgery, the incidence is as great as 50 to $79 \%[3,4]$. There are no significant differences in the frequency of nausea based on the craniotomy location [5]. PONV has a multi-factor etiology and the mechanism is complex. Currently, pharmacological prophylaxis is widely used in clinical practice, and the most commonly used prophylactic antiemetics include serotonin $\left(5-\mathrm{HT}_{3}\right)$ receptor antagonists often in combination with either droperidol or dexamethasone [6,7]. Although droperidol 
is very effective, it can cause death secondary to arrhythmia or QT prolongation [8]. At present, no therapy is absolutely effective at preventing PONV.

Due to the limited efficacy and many side effects of drug therapy, various non-pharmacological techniques have been used in clinical practice. These therapies include acupuncture, acupressure, transcutaneous electrical nerve stimulation (TENS), electro-acupuncture, and others. Early in 2006, the American Society of PeriAnesthesia Nurses (ASPAN) recommended pericardium 6 (P6; also known as Neiguan) acupoint stimulation (Class IIb, Level A) as a complementary intervention for PONV prophylaxis [9]. According to the theory of traditional Chinese medicine (TCM), performing surgery breaks the balanced state of the human body and disturbs the movement of both $q i$ and blood. When this happens, stomach qi will reverse its direction and go upward, causing nausea and vomiting. The main principle of treatment in this case is to regulate the function of the stomach to avoid the adverse flow of qi. P6 is an acupuncture point in the meridian named Jueyin Pericardium Meridian of Hand. It is both the Luoconnecting point (the point where a collateral starts to connect a definite pair of Yin and Yang meridians, which are externally-internally related) and the eight confluent point (the point connecting the eight extra meridians with the twelve primary meridians). One of P6's main functions is to regulate the function of the stomach to avoid the adverse flow of $q i$, thus it is an effective acupoint for preventing nausea and vomiting.

Many recent studies have supported the efficacy of P6 acupoint stimulation for preventing PONV [10-12], with very few gaining a negative result $[13,14]$. The various ways to stimulate the P6 acupoint include laser, electrical, transcutaneous electrical and manual stimulation. Few studies have compared the efficacy of invasive methods with non-invasive methods. Thus, the optimal method of stimulation has not been determined due to lack of evidence. Although there is a great amount of research concerning P6 acupoint stimulation for preventing PONV, only two studies focus on the role of P6 acupoint stimulation in postoperative patients following craniotomy $[15,16]$. Therefore, we designed a single-center, prospective, double-blind, five-arm, parallel-group, randomized controlled trial (RCT) to evaluate the efficacy of P6 acupoint stimulation as a non-pharmacological prophylaxis for PONV.

The primary objective of this study is to evaluate the efficacy of P6 acupoint stimulation versus placebo, and versus routine antiemetic for the prevention of PONV in patients undergoing craniotomy under general anesthesia. The secondary study objective is to compare the efficacy of invasive acupuncture with non-invasive TENS on P6. We hypothesize that a significantly greater percentage of patients will not vomit in the subsequent 24 hours following craniotomy in the acupoint stimulation groups. Additionally, we expect that the severity of nausea will be decreased in the acustimulation groups, based on subjective patient feedback.

\section{Methods/design \\ Design}

This is a single-center, prospective, double-blind, five-arm, parallel-group, RCT. The trial protocol strictly follows the principles of the Declaration of Helsinki (version Seoul, 2008) and approval has been obtained from Si-chuan University's Ethics and Research Committee. Participants have been and will continue to be recruited from the West China Hospital of Si-chuan University (WCHSU) from January 2013 to October 2013 (Figure 1). All participants are required to give written informed consent.

\section{Patient population and setting}

A total of 300 Chinese patients undergoing craniotomy will be sequentially recruited at the WCHSU after fulfilling the eligibility criteria and signing the informed consent. A clinical assistant with institutional review board training will be in charge of patient enrollment.

\section{Eligibility criteria \\ Inclusion criteria}

Patients who fulfill the following conditions will be included: 1) scheduled for neurosurgery requiring opening of the cranium and dura; 2) aged between 18 and 70 years old; 3) American Society of Anesthesiologists (ASA) physical status classification of I or II; 4) undergoing general anesthesia; 5) no history of PONV or motion sickness; 6) no use of antiemetic 24 hours before surgery; 7) willing to participate; 8) no experience with acupuncture therapies; and 9) signed an informed consent form.

\section{Exclusion criteria}

Participants that meet any of the following criteria will be excluded: 1) nausea or vomiting 24 hours before surgery; 2) pregnant or lactating women; 3) drug or alcohol abusers; 4) recipients of chemotherapy or radiation therapy during the previous 7 days; 5) cardiac pacemaker; 6) menstruating phase of the menstrual cycle; 7) refusal to accept acupuncture and TENS treatment; 8) mental disorder; 9) history of epilepsy and still taking an antiepileptic medicine; 10) unconscious before the surgery; 11) cannot normally communicate; 12) undergoing ventricle or brainstem surgery; 13) cerebral perfusion pressure (CPP) of less than $50 \mathrm{mmHg}$ or greater than 150 $\mathrm{mmHg}$; 14) poorly controlled diabetes mellitus (fasting plasma glucose greater than $12 \mathrm{mmol} / \mathrm{L}) ; 15)$ bleeding disorders (hemophilia or afibrinogenemia); and 16) serious systemic disease (AIDS or sepsis). 


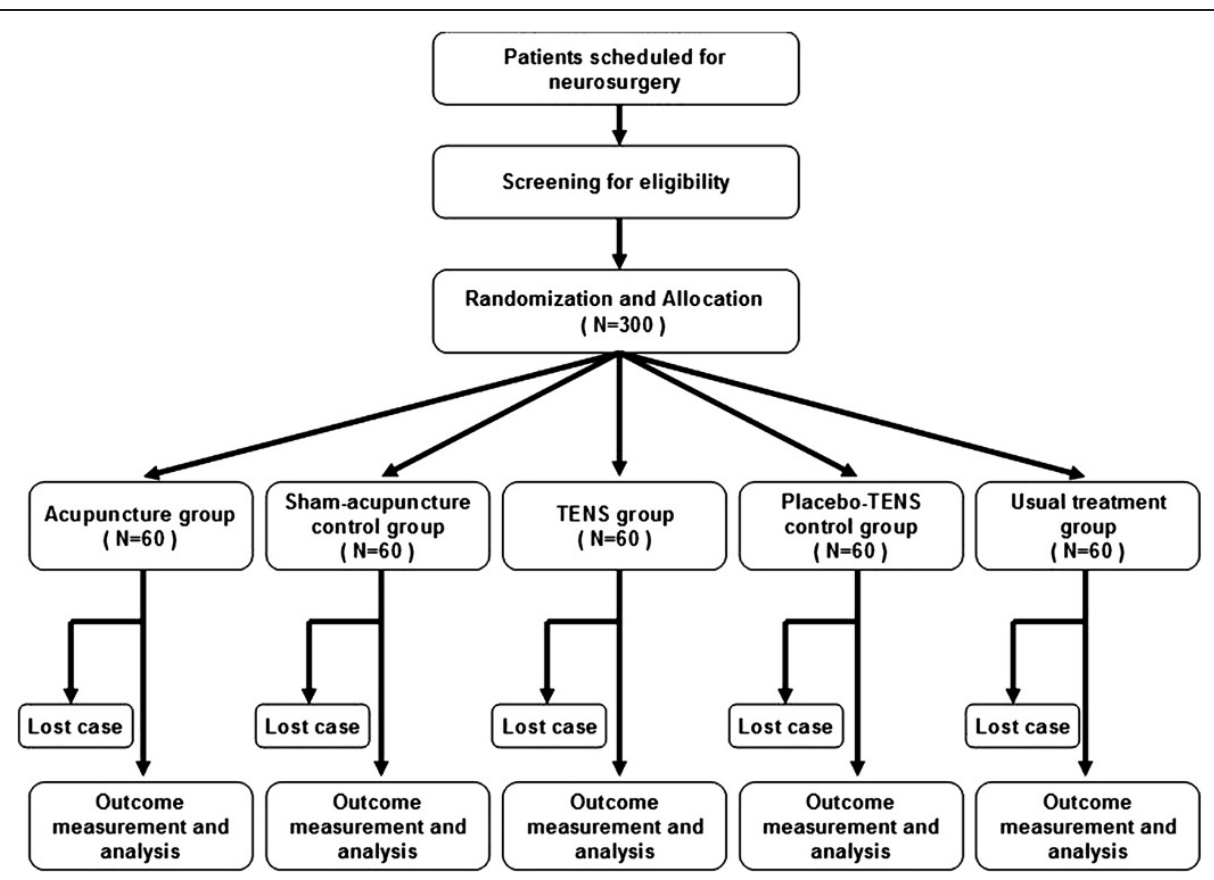

Figure 1 Trial flow chart. If a patient can not complete the study due to death, re-operation, unconsciousness or cognitive impairment, the patient will be considered a lost case and recorded as an occurrence of PONV. PONV, postoperative nausea and vomiting; TENS, transcutaneous electrical nerve stimulation.

\section{Randomization and blinding}

The randomization sequence will be computer-generated by independent research staff using software called Package for Encyclopaedia Medical Statistics 3.1 (PEMS 3.1). Stratification will not be used in this trial. After randomization, group assignments will be concealed in lightproof and sealed envelopes. The included participants will be randomly enrolled by sealed envelope and assigned to the acupuncture group, sham-acupuncture control group, TENS group, placebo-TENS control group and usual treatment group, using a 1:1:1:1:1 ratio. Patient allocation will be performed by a clinical assistant, who will be trained in institutional review board policies. All study personnel including the outcome assessors, study participants, data analyst and care providers will be blinded to group assignments.

\section{Interventions}

In this trial, we will use both invasive and non-invasive methods to stimulate the P6 acupoint as an adjunct to routine antiemetic drug therapy for the prevention of nausea and vomiting in patients undergoing craniotomy. The routine antiemetic treatment for high-risk patients at the WCHSU is a single intravenous dose of $8 \mathrm{mg}$ ondansetron administered before skin closure. Therefore, neither dexamethasone nor droperidol will be used in our study.

To date, the optimal timing of acustimulation for preventing PONV has not been determined. In our study, the intervention is started right after the patient regains consciousness from anesthesia for two reasons. First, in the TCM theory, it is important to locate the right acupoint with the de qi sensation (the sensation of numbness, heaviness or distention associated with correct identification of an acupuncture point) to enhance efficacy, and this cannot be accomplished when the patient is unconscious. Second, a recent RCT shows that postoperative acustimulation is more effective in reducing PONV [17].

In preparation for surgery, all patients will receive a standard anesthesia and analgesia regimen specific to neurosurgical procedures. They will fast for 8 hours before general anesthesia. Standard monitors will be used to record patients' electrocardiogram, pulse oximetry, invasive arterial blood pressure and end tidal $\mathrm{CO}_{2}$. Induction of anesthesia will be achieved with midazolam $0.05 \mathrm{mg} / \mathrm{kg}$, sufentanil $0.3 \mu \mathrm{g} / \mathrm{kg}$, atracurium $0.15 \mathrm{mg} / \mathrm{kg}$ and propofol $2 \mathrm{mg} / \mathrm{kg}$. When endotracheal intubation and gastrointestinal decompression with either an orogastric or nasogastric tube are undertaken, the anesthesia will be maintained with $50 \%$ nitrous oxide and $3 \%$ sevoflurane, and intermittent sufentanil $0.2 \mu \mathrm{g} / \mathrm{kg}$ and atracurium $0.1 \mathrm{mg} / \mathrm{kg}$. Intravenous ondansetron $8 \mathrm{mg}$ is administered as a routine antiemetic treatment for each patient before skin closure. Fentanyl 25 to $50 \mu \mathrm{g}$ intravenous boluses are used as needed for postoperative, patient-controlled intravenous analgesia (PCIA). After surgery, patients will be continually monitored in the post-anesthesia care unit (PACU). Tracheal extubation will be undertaken when patients regain consciousness from anesthesia. Intramuscular me- 
toclopramide $10 \mathrm{mg}$ will be used as a standard rescue antiemetic and administered at the patient's request.

When the patients regain consciousness from anesthesia and are able to communicate, the intervention will begin. All participants in our study will receive the intervention only once. In preparation for acustimulation, the patient will be placed in the best posture for P6 acupuncture, with their forearms fully exposed and with their palms facing upward. A fourth-year acupuncture resident will locate the P6 acupoint bilaterally (Figure 2) and perform the interventions specified in Table 1. P6 is an important acupuncture point in the Jueyin Pericardium Meridian of Hand. The location of P6 is 2 cun (a cun is a measurement used in acupuncture and is equivalent to the width of the distal interphalangeal joint of the first finger) above the transverse crease of the wrist, between the tendons of palmaris longus and flexor carpi radialis.

For the acupuncture group (group A), after skin cleaning with $75 \%$ alcohol swab, sterile and disposable stainless steel needles $(0.25 \times 25 \mathrm{~mm}$, Wuxi Jiajian, Jiangsu, China) are quickly and perpendicularly inserted into the skin at P6 acupoints bilaterally to a depth of 20 $\mathrm{mm}$. In this group, downward pressure and upward lifting combined with twirling the needle will be used to achieve de qi sensation. The needles will be kept in place for 30 minutes and manipulated manually every 10 minutes to maintain the de qi sensation. When the treatment time is over, all needles will be carefully taken out and the puncture sites will be covered with sterile swabs to avoid bleeding.

For the sham-acupuncture control group (group SA), sham points are used, which are superficial, nonacupoints on the radial side of each wrist, $15 \mathrm{~mm}$ away from each P6 acupoint (Figure 2). After skin cleaning with

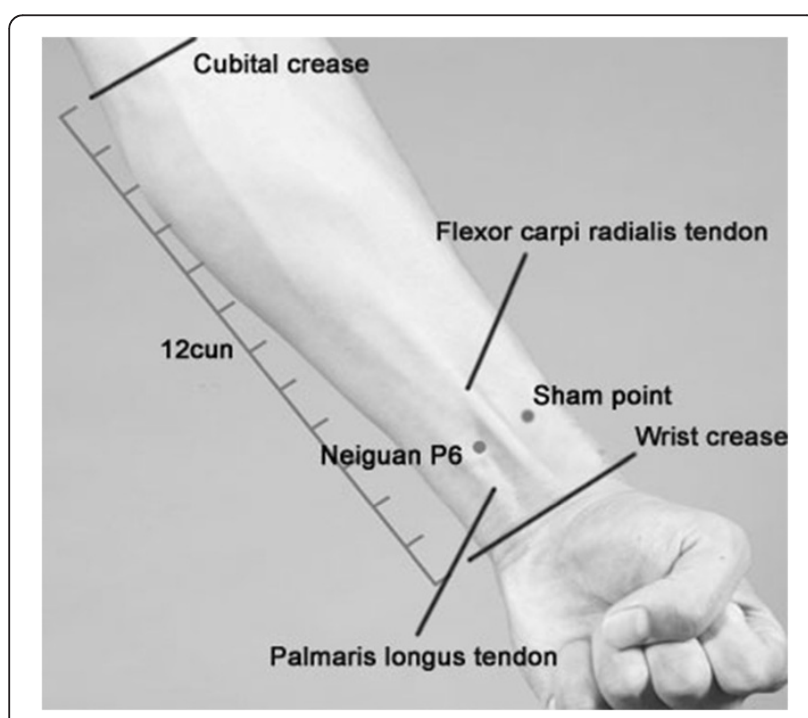

Figure 2 Location of P6 acupoint and sham acupoint.
$75 \%$ alcohol swab, sterile and disposable stainless steel needles $(0.25 \times 25 \mathrm{~mm}$, Wuxi Jiajian, Jiangsu, China) are quickly and perpendicularly inserted into the skin at sham acupoints bilaterally to a depth of $5 \mathrm{~mm}$. The de qi sensation is not required in this group. The needles will be retained for 30 minutes as with the group A, but will not be stimulated or manipulated for the duration. After 30 minutes, the same method will be used to take out the needles as in the group A.

For the TENS group (group T), the patients' forearms are cleaned with $75 \%$ alcohol swabs and self-adhesive electrodes are placed over P6 acupoints bilaterally. SDZ-V nerve and muscle stimulators (Hwato, Jiangsu, China) are used for TENS (Figure 3). The treatment time is also 30 minutes, using dilatational wave. The stimulation frequency is set on 2 to 100 hertz and intensity varies until the patients feel the de qi sensation continuously. The screen on each stimulator is covered with $6 \times 8 \mathrm{~cm}$ black tape so that no one will see the screen and know whether the stimulator is on or off. When the treatment time is complete, all self-adhesive electrodes will be removed.

For the placebo-TENS control group (group PT), patients have self-adhesive electrodes placed over P6 acupoints bilaterally, and are connected to the SDZ-V nerve and muscle stimulators for 30 minutes. However, as a placebo control group, the stimulators will not be turned on and there will be no electrical stimulation during the treatment. In order to blind patients in this group, each participant will be informed that special acupoint stimulation is used in the treatment which cannot be felt by human sensory perception. After $30 \mathrm{mi}$ nutes, all self-adhesive electrodes will be removed as in the group $\mathrm{T}$.

For the usual treatment group (group U), no interventions are undertaken. Patients in this group will be observed by the researchers as a blank control, which will provide a baseline postoperative vomiting rate and nausea score in this study using the same drug prophylaxis.

\section{Outcome measurement}

In our study, outcomes are collected based on previously designed PONV studies [18]. The whole study period covers 24 hours after the surgery. A separate research nurse, who is not involved in the management of patients, will record anesthesia time, surgery time, endotracheal intubation time, patient demographics and preoperative data for each patient. Demographic and preoperative data will include: age, gender, race, weight, height, acupuncture experience, smoking history and CPP. Another blinded observer will record the postoperative data, which will include administration of a rescue antiemetic, patient satisfaction with PONV management, and episodes of vomiting or nausea at 2 hours, 6 hours and 24 hours postoperatively. The physicians will record the use time and 
Table 1 Details of interventions

\begin{tabular}{|c|c|c|c|}
\hline Group names & Acupoints & $\begin{array}{l}\text { De qi } \\
\text { sensation }\end{array}$ & Interventions \\
\hline $\begin{array}{l}\text { Acupuncture group } \\
\text { (group A) }\end{array}$ & $\begin{array}{l}\text { P6 (Neiguan) } \\
\text { bilaterally }\end{array}$ & Yes & $\begin{array}{l}\mathrm{P} 6 \text { at both sides is punctured perpendicularly to a depth of } 20 \mathrm{~mm} \text {. Needles are retained for } 30 \\
\text { minutes and stimulated every } 10 \text { minutes to keep the de qi sensation. }\end{array}$ \\
\hline $\begin{array}{l}\text { Sham-acupuncture } \\
\text { control group } \\
\text { (group SA) }\end{array}$ & $\begin{array}{l}\text { Sham points } \\
\text { bilaterally }\end{array}$ & No & $\begin{array}{l}\text { The sham point is punctured to a perpendicular depth of } 5 \mathrm{~mm} \text { bilaterally. Needles are retained } \\
\text { for } 30 \text { minutes and no stimulation is performed during this duration. }\end{array}$ \\
\hline $\begin{array}{l}\text { TENS group } \\
(\text { group } T)\end{array}$ & $\begin{array}{l}\text { P6 (Neiguan) } \\
\text { bilaterally }\end{array}$ & Yes & $\begin{array}{l}\text { P6 acupoint at both sides is stimulated by active self-adhesive electrodes for } 30 \text { minutes. The } \\
\text { stimulation frequency is set on } 2 \text { to } 100 \text { hertz and intensity varies until the patients continue to } \\
\text { feel the de qi sensation. }\end{array}$ \\
\hline $\begin{array}{l}\text { Placebo-TENS control } \\
\text { group } \\
\text { (group PT) }\end{array}$ & $\begin{array}{l}\text { P6 (Neiguan) } \\
\text { bilaterally }\end{array}$ & No & Inactive self-adhesive electrodes are placed at the P6 acupoint bilaterally for 30 minutes. \\
\hline $\begin{array}{l}\text { Usual treatment } \\
\text { group } \\
\text { (group U) }\end{array}$ & - & - & - \\
\hline
\end{tabular}

TENS, transcutaneous electrical nerve stimulation.

dosage of rescue antiemetic for each patient when they request rescue therapy, and the records will be handed over to the observer for assessment. Nausea is a subjective sensation which should be evaluated by the patient, not by the observer. Thus, the observer will request the patients to evaluate their nausea score using a standard visual analogue scale (VAS) (0, no nausea at all; 10 , worst

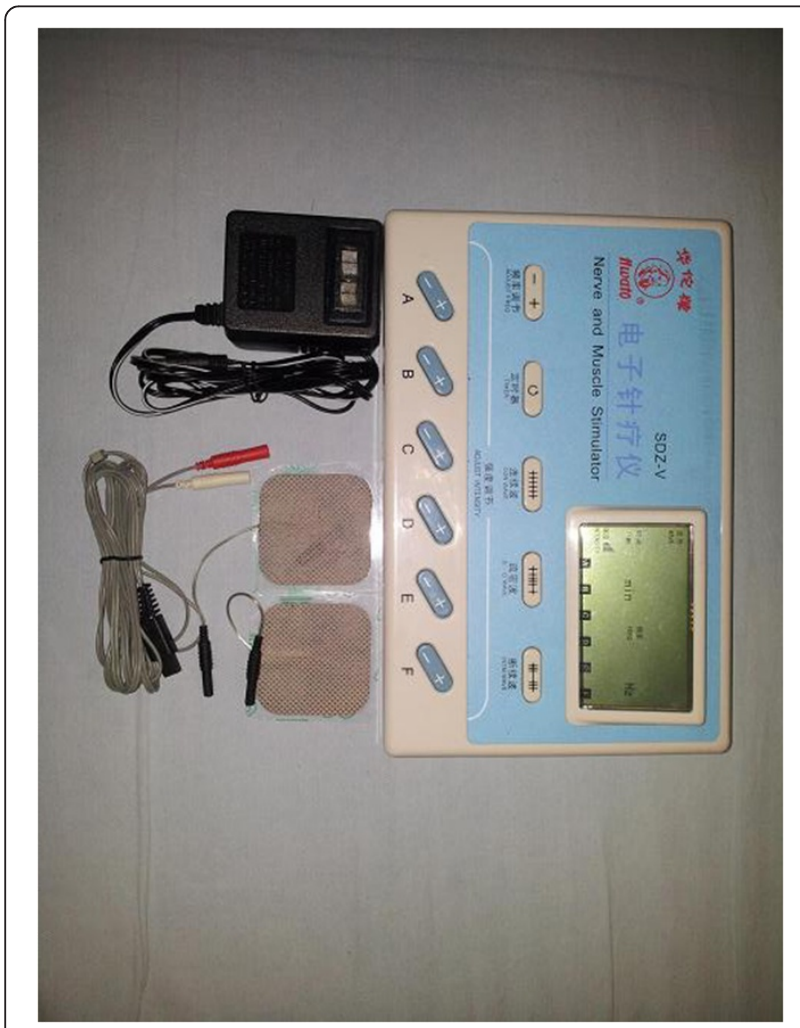

Figure 3 The SDZ-V nerve and muscle stimulator (Hwato, Jiangsu, China) and self-adhesive electrodes. imaginable nausea). Vomiting, which is defined as the ejection of contents of the stomach through the mouth, will be reported by the patients and assessed by this blinded observer. Patient satisfaction is not an easy concept to measure. Until recently, there has been no consensus on how to measure this concept. Since satisfaction is multi-faceted and subjective, a questionnaire cannot cover every aspect of a patient's concerns. Therefore, in this study, patients will be asked about satisfaction with their PONV treatment at 24 hours postoperatively using another 0 (very dissatisfied) to 10 (very satisfied) scale.

The incidence of postoperative vomiting within 24 hours after craniotomy across the five groups is the main outcome we are studying. The secondary outcomes are: 1 ) proportion of participants with a complete response (no vomiting, no nausea, no rescue antiemetic) during 0 to 2 hours, 2 to 6 hours, 6 to 24 hours and 0 to 24 hours after craniotomy under general anesthesia; 2) assessment of nausea score (severity of nausea) during 0 to 2 hours, 2 to 6 hours and 6 to 24 hours after craniotomy under general anesthesia; 3) assessment of total rescue antiemetic dosage during 0 to 24 hours after craniotomy under general anesthesia; and 4) assessment of satisfaction score (patient satisfaction with PONV management) at 24 hours postoperatively.

\section{Sample size calculation and statistical analysis}

A German prospective observational study published in 2011 demonstrated an overall incidence of PONV in 47\% of patients after craniotomy under general anesthesia [4]. The sample size is determined by using PEMS 3.1 with $\alpha=0.05$ (two-sided) and $\beta=0.1$ (90\% power). In order to demonstrate a $30 \%$ absolute reduction in the incidence of PONV, the sample size will be 49 patients for each group. Considering the potential loss and attrition, 60 patients 
per group, or a total of 300 patients, should be reasonable. If a patient can not complete the study due to death, reoperation, unconsciousness or cognitive impairment, the patient will be considered a lost case and recorded as an occurrence of PONV.

All data will be analyzed by a blinded statistician using PEMS 3.1 at a separate location from the WCHSU. The intention-to-treat principal will be used in the data analysis. Baseline data will be collected and compared first. Different statistics are presented differently, for example, continuous data is presented as mean ( \pm standard deviation). Chi-square test is used to compare the incidence of postoperative vomiting, complete response rate, sex difference and other nominal data. Conversely, the Kruskal-Wallis test is used to compare the nausea score, satisfaction score and antiemetic dosage. Additionally, the Nemenyi test and Scheffés method are used for multiple comparisons between groups. A $P$ value $<0.05$ is considered statistically significant.

\section{Discussion}

This study could potentially confirm that P6 acupoint stimulation is an effective adjunct to the standard antiemetic drug therapy for the prevention of PONV in patients undergoing craniotomy. Our study may also confirm that conventional acupuncture is more effective than TENS. The reason why we chose 2 hours, 6 hours and 24 hours postoperatively as the observation points are because: 1) volatile anesthetics may be the main cause for early (within 2 hours after the surgery) but not delayed postoperative nausea and vomiting [19]; 2) the half-life of ondansetron is approximately 6 hours based on pharmacokinetics [20]; and 3) PONV is defined as vomiting and/ or nausea occurring within 24 hours after surgery [21].

Previous studies have not indicated whether the duration of P6 acupoint stimulation alters its effect on PONV. From our point of view, a long duration of acupoint stimulation will increase patients' discomfort and pain, especially for those in the acupuncture group. However, if the stimulation time is too short, the therapeutic effect of acupuncture on PONV will be uncertain. In our study, we believe the most reasonable duration of P6 acupoint stimulation is 30 minutes, which is the common acupuncture treatment time at WCHSU.

The most difficult part in this research will be to blind the patients in different treatment groups. Recruiting patients with no experience of any kind of acupuncture therapies is part of the basic method, since patients who have not experienced de qi sensation are easier to blind. Furthermore, different sham groups are set up according to different stimulation methods. Acupuncture is an invasive method and we chose to blind the patients by superficially acupuncturing the sham point, which has been commonly used as an invasive control condition in several studies
[22-24]. TENS is used as the non-invasive method and we chose to blind the patients by using inactive self-adhesive electrodes. In order to better blind the patients using the non-invasive methods, each participant will be informed that special acupoint stimulation is used in the treatment which cannot be felt by human sensory perception.

\section{Trial status}

This trial is currently recruiting participants.

\section{Abbreviations}

ASA: American Society of Anesthesiologists; ASPAN: American Society of PeriAnesthesia Nurses; CPP: Cerebral perfusion pressure; P6: Pericardium 6; PACU: Post-anesthesia care unit; PCIA: Patient-controlled intravenous analgesia; PEMS: Package for Encyclopaedia Medical Statistics; PONV: Postoperative nausea and vomiting; RCT: Randomized controlled trial; TCM: Traditional Chinese medicine; TENS: Transcutaneous electrical nerve stimulation; VAS: Visual analogue scale; WCHSU: West China Hospital of Si-chuan University.

\section{Competing interests}

The authors declare that they have no competing interests.

\section{Authors' contributions}

J-QL, R-ZF and NL all contributed to the design and development of the study protocol. J-QL prepared the initial draft of the manuscript. NL was the general supervisor for this research. All authors critically reviewed the content and approved the final version.

\section{Acknowledgments}

We would like to thank Angela K Johnson and Juan D Montoya from the University of Toledo, College of Medicine and Life Sciences, Toledo, OH, USA, for their support in reviewing and revising our manuscript.

Received: 1 February 2013 Accepted: 14 May 2013

Published: 28 May 2013

\section{References}

1. Gan TJ, Meyer T, Apfel CC, Chung F, Davis PJ, Eubanks S, Kovac A, Philip BK, Sessler DI, Temo J, Tramer MR, Watcha M: Consensus guidelines for managing postoperative nausea and vomiting. Anesth Analg 2003, 97:62-71.

2. Apfel CC, Kranke P, Eberhart LH: Comparison of surgical site and patient's history with a simplified risk score for the prediction of postoperative nausea and vomiting. Anaesthesia 2004, 59:1078-1082.

3. Apfel CC, Laara E, Koivuranta M, Greim CA, Roewer N: A simplified risk score for predicting postoperative nausea and vomiting: conclusions from cross-validations between two centers. Anesthesiology 1999, 91:693-700.

4. Latz B, Mordhorst C, Kerz T, Schmidt A, Schneider A, Wisser G, Werner C, Engelhard $K$ : Postoperative nausea and vomiting in patients after craniotomy: incidence and risk factors. J Neurosurg 2011, 114:491-496.

5. Irefin SA, Schubert A, Bloomfield EL, DeBoer GE, Mascha EJ, Ebrahim ZY: The effect of craniotomy location on postoperative pain and nausea. J Anesth 2003, 17:227-231.

6. Sanchez-Ledesma MJ, Lopez-Olaondo L, Pueyo FJ, Carrascosa F, Ortega A: A comparison of three antiemetic combinations for the prevention of postoperative nausea and vomiting. Anesth Analg 2002, 95:1590-1595.

7. Habib AS, El-Moalem HE, Gan TJ: The efficacy of the 5-HT3 receptor antagonists combined with droperidol for PONV prophylaxis is similar to their combination with dexamethasone. A meta-analysis of randomized controlled trials. Can J Anaesth 2004, 51:311-319.

8. Charbit B, Albaladejo P, Funck-Brentano C, Legrand M, Samain E, Marty J: Prolongation of QTc interval after postoperative nausea and vomiting treatment by droperidol or ondansetron. Anesthesiology 2005, 102:1094-1100.

9. American Society of PeriAnesthesia Nurses PONV/PDNV Strategic Work Team: ASPAN'S evidence-based clinical practice guideline for the 
prevention and/or management of PONV/PDNV. J Perianesth Nurs 2006, 21:230-250.

10. Lee A, Fan LT: Stimulation of the wrist acupuncture point $P 6$ for preventing postoperative nausea and vomiting. Cochrane Database Syst Rev 2009:CD003281.

11. Gan TJ, Jiao KR, Zenn M, Georgiade G: A randomized controlled comparison of electro-acupoint stimulation or ondansetron versus placebo for the prevention of postoperative nausea and vomiting. Anesth Analg 2004, 99:1070-1075.

12. El-Deeb AM, Ahmady MS: Effect of acupuncture on nausea and/or vomiting during and after cesarean section in comparison with ondansetron. J Anesth 2011, 25:698-703.

13. Habib AS, Itchon-Ramos N, Phillips-Bute BG, Gan TJ: Transcutaneous acupoint electrical stimulation with the ReliefBand for the prevention of nausea and vomiting during and after cesarean delivery under spinal anesthesia. Anesth Analg 2006, 102:581-584

14. Agarwal A, Pathak A, Gaur A: Acupressure wristbands do not prevent postoperative nausea and vomiting after urological endoscopic surgery. Can J Anaesth 2000, 47:319-324.

15. Wang $X Q$, Yu JL, Du ZY, Xu R, Jiang CC, Gao X: Electroacupoint stimulation for postoperative nausea and vomiting in patients undergoing supratentorial craniotomy. J Neurosurg Anesthesiol 2010, 22:128-131.

16. Xu M, Zhou SJ, Jiang CC, Wu Y, Shi WL, Gu HH, Cai HD, Wang XQ: The effects of P6 electrical acustimulation on postoperative nausea and vomiting in patients after infratentorial craniotomy. J Neurosurg Anesthesiol 2012, 24:312-316.

17. White PF, Hamza MA, Recart A, Coleman JE, Macaluso AR, Cox L, Jaffer O, Song D, Rohrich R: Optimal timing of acustimulation for antiemetic prophylaxis as an adjunct to ondansetron in patients undergoing plastic surgery. Anesth Analg 2005, 100:367-372.

18. Apfel CC, Roewer N, Korttila K: How to study postoperative nausea and vomiting. Acta Anaesthesiol Scand 2002, 46:921-928.

19. Apfel CC, Kranke P, Katz MH, Goepfert C, Papenfuss T, Rauch S, Heineck R, Greim CA, Roewer N: Volatile anaesthetics may be the main cause of early but not delayed postoperative vomiting: a randomized controlled trial of factorial design. Br J Anaesth 2002, 88:659-668.

20. Pritchard JF, Bryson JC, Kernodle AE, Benedetti TL, Powell JR: Age and gender effects on ondansetron pharmacokinetics: evaluation of healthy aged volunteers. Clin Pharmacol Ther 1992, 51:51-55.

21. McCracken G, Houston P, Lefebvre G: Guideline for the management of postoperative nausea and vomiting. J Obstet Gynaecol Can 2008, 30:600-607. 608-616.

22. Crew KD, Capodice JL, Greenlee H, Brafman L, Fuentes D, Awad D, Yann Tsai W, Hershman DL: Randomized, blinded, sham-controlled trial of acupuncture for the management of aromatase inhibitor-associated joint symptoms in women with early-stage breast cancer. J Clin Oncol 2010, 28:1154-1160.

23. Endres $\mathrm{HG}$, Bowing G, Diener HC, Lange S, Maier C, Molsberger A, Zenz M, Vickers AJ, Tegenthoff M: Acupuncture for tension-type headache: a multicentre, sham-controlled, patient-and observer-blinded, randomised trial. J Headache Pain 2007, 8:306-314.

24. Carlsson CP, Axemo P, Bodin A, Carstensen H, Ehrenroth B, Madegard-Lind I, Navander C: Manual acupuncture reduces hyperemesis gravidarum: a placebo-controlled, randomized, single-blind, crossover study. J Pain Symptom Manage 2000, 20:273-279.

doi:10.1186/1745-6215-14-153

Cite this article as: Lv et al.: P6 acupoint stimulation for prevention of postoperative nausea and vomiting in patients undergoing craniotomy: study protocol for a randomized controlled trial. Trials 2013 14:153.

\section{Submit your next manuscript to BioMed Central and take full advantage of:}

- Convenient online submission

- Thorough peer review

- No space constraints or color figure charges

- Immediate publication on acceptance

- Inclusion in PubMed, CAS, Scopus and Google Scholar

- Research which is freely available for redistribution

Submit your manuscript at www.biomedcentral.com/submit
Ciomed Central 